\title{
Feasibility of an EUS e-training course with live cases $\square$
}

\section{(2) $\odot \Theta$}

Authors

Vinay Dhir, Priyanka Udawat, Rahul Shah, Aruna Alahari

\section{Institution}

Institute of Digestive and Liver Care, SI Raheja Hospital School of EUS, Mumbai, India

submitted 19.2.2021

accepted after revision 22.3.2021

Bibliography

Endosc Int Open 2021; 09: E1291-E1296

DOI 10.1055/a-1482-8303

ISSN 2364-3722

(C) 2021. The Author(s).

This is an open access article published by Thieme under the terms of the Creative Commons Attribution-NonDerivative-NonCommercial License, permitting copying and reproduction so long as the original work is given appropriate credit. Contents may not be used for commercial purposes, or adapted, remixed, transformed or built upon. (https://creativecommons.org/licenses/by-nc-nd/4.0/)

Georg Thieme Verlag KG, Rüdigerstraße 14,

70469 Stuttgart, Germany

Corresponding author

Vinay Dhir, Institute of Digestive and Liver Care Gastroenterology, SI Raheja Hospital, Mahim, Mumbai Maharashtra 400016, India

Fax: + 912222804769

vinaydhir@gmail.com

\section{ABSTRACT}

Background and study aims One-on-one endoscopic ultrasound (EUS) mentorship was not possible at most institutions during the COVID-19 pandemic. We decided to test the feasibility of structured training in EUS with virtual eclasses with live cases. The aim of this study was to assess the feasibility of a virtual EUS training course with objective end points.

Patients and methods Twenty-one trainees were trained on a virtual platform over 16 classes of 90 minutes each, over 3 months. The virtual training screen had two equal parts, one showing the endoscopist's hand movements, and another the resultant EUS display. The course curriculum included EUS anatomy of the mediastinum, pancreatico-biliary region and rectum. The assessment was done on videos of procedures performed by trainees.

Results Twenty trainees performed 251 EUS procedures (range $8-25$, mean $12.5 \pm 4.9$ ) at their institutions. At the end of the course, all students $(100 \%)$ could maneuver the echo-endoscope through to the duodenum. Fifteen trainees sent a video for final assessment. The successful specified area identification rates were 12 of 15 (80\%) for the subcarinal space, 10 of15 (66.6\%) for the head of the pancreas, 10 of $15(66.6 \%)$ for the common bile duct, and nine of $15(60 \%)$ for the tail of pancreas. The success rate of ability to get appropriate windows was 10 of 15 (67\%) for the subcarinal space, eight of 15 (53.3\%) for the head of the pancreas, seven of 15 (46.6\%) for the common bile duct and six of 15 (40\%) for the tail of pancreas. No adverse events were reported.

Conclusions A virtual EUS training course with live cases appears feasible. It allows the possibility of training a large number of students. Further evaluation is needed, especially of virtual assessment methods and training benchmarks.

\section{Introduction}

Endoscopic ultrasound (EUS) is established as a modality for the diagnosis and management of pancreato-biliary and gastrointestinal disorders. EUS requires both cognitive and technical skills for adequate evaluations. The requirement for trained professionals is increasing worldwide. The American Society for Gastrointestinal Endoscopy (ASGE) recommends that at least 225 hands-on EUS cases be performed before competency can be assessed [1]. There are several methods to acquire theoretical knowledge through lectures, textbooks, atlases, slides, videos and websites. Practical EUS training is acquired in ad- vanced fellowship programs, EUS-specific seminars with live endoscopy workshops, and hands-on training using animal or phantom models. One-on-one hands-on mentorship during fellowship is the most appropriate method for EUS training. The availability of fellowships with hands-on training is limited. This creates a demand for EUS training outside of advanced fellowships. A structured virtual EUS training program with live procedures has not been evaluated before. Virtual training may offer the advantage of live visual details of the echo-endoscope maneuvers, simultaneously with resultant ultrasound images. It also allows the possibility of repeating the maneuver on demand and two-way communications between the teacher 
- Table 1 Details about trainees.

\begin{tabular}{|c|c|}
\hline Total number of trainees $(n)$ & 21 \\
\hline \multicolumn{2}{|l|}{ Gender } \\
\hline - Male & 14 \\
\hline - Female & 7 \\
\hline \multicolumn{2}{|l|}{ Years since gastroenterology degree/ fellowship } \\
\hline - Nil & 1 \\
\hline - $1-5$ years & 19 \\
\hline - >5 years & 1 \\
\hline \multicolumn{2}{|l|}{ Current affiliation } \\
\hline - Academic/university & 8 \\
\hline - Private hospital & 13 \\
\hline \multicolumn{2}{|l|}{ ERCP experience (performed ERCP independently) } \\
\hline - Yes & 20 \\
\hline - No & 1 \\
\hline \multicolumn{2}{|l|}{ Prior experience (EUS) } \\
\hline - $<50$ procedures & 4 \\
\hline $\begin{array}{l}\text { - Only observation (conferences/in vitro/hands-on } \\
\text { training on models) }\end{array}$ & 14 \\
\hline - Only internet (videos) & 3 \\
\hline $\begin{array}{l}\text { ERCP, endoscopic retrograde cholangiopancreatography; } \\
\text { ultrasound. }\end{array}$ & copic \\
\hline
\end{tabular}

and trainee. In addition, a large number of trainees can learn basic EUS skills on the same patient.

During the recent COVID-19 pandemic-induced restrictions worldwide, mentorship was not possible at most places. After resumption of endoscopy services, we decided to assess the feasibility of a virtual EUS training course by utilizing e-classes with live cases with objective end points for satisfactory identification of specific structures, and for learning basic maneuvers.

\section{Patients and methods}

This was a prospective observational study. Twenty-one trainees were chosen from a group of applicants. The criteria for choosing the candidate were: 1 . at least 3 years' experience in gastroscopy and colonoscopy with certification; 2 . optional exposure to EUS and ERCP; 3. availability of linear echo-endoscope at the candidate's institute; and 4. access to high-speed internet. Details of trainees are given in $>$ Table 1. Applicants who already were well trained in EUS were not considered. The training was imparted over a virtual platform. Sixteen classes of 90 minutes each were held over 3 months (August 1, 2020 to October 31, 2020). Twenty-one patients with appropriate indications (esophageal cancers, mediastinal lymphadenopathy, abdominal lymphadenopathy, gastric wall lesion, pancreatic le-
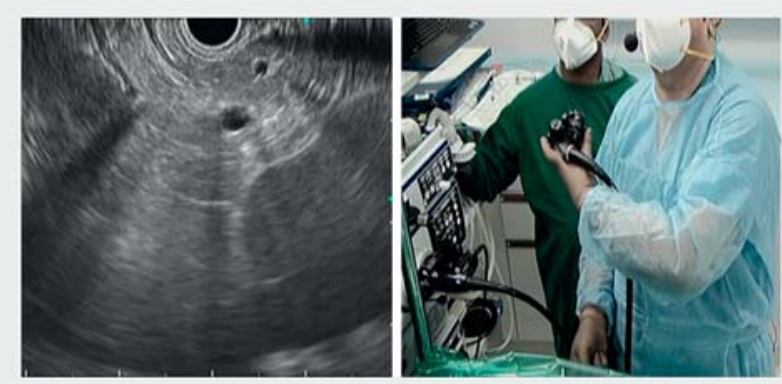

Fig. 1 Virtual training screen. Two equal images, one image showing examiner's hand movements with echo-endoscope and the other image shows the corresponding EUS picture.

sion, bile duct lesion, rectal lesion etc.) were included for the purpose of training. Informed consent was taken from patients.

Patients with cardiorespiratory abnormality, bleeding diathesis or other risk factors for anesthesia were not included. During the procedure, patients were sedated using propofol by a senior anesthetist. Continuous cardiorespiratory monitoring was done with availability of an anesthesia work station (Datex-Ohmeda) and defibrillator. Procedures were done by a single endoscopist with more than 20 years of experience in diagnostic and therapeutic EUS. A linear echo-endoscope was used for all upper gastrointestinal procedures (Olympus GF-UCT 140, Olympus Inc., Tokyo). A radial echo-endoscope was used for rectal anatomy (Olympus GF-UE 160). Fine-needle biopsy (FNB) was demonstrated with an Acquire 22G needle (Boston Scientific, Massachusetts, United States).

All patients underwent a COVID (reverse transcription polymerase chain reaction) test within 48 hours prior to the procedure. Any patient with cough, fever, or any other symptoms on the day of endoscopy was not included. All endoscopy staff members in the operating room wore N-95 masks.

Our endoscopy rooms are equipped with built-in two-way audiovisual transmission and procedures can be live-streamed to any part of the world, accompanied by discussion in real time. It is possible to transmit simultaneously external camera, EUS, \& X ray Images. Zoom platform (zoom.us, San Jose, California, United States) was used for the live transmission. The virtual training screen consisted of two equal- sized images, one showing the examiner's hand movements with echo-endoscope and another, the EUS display. Either image could be further magnified if needed. The images were in real time and were projected simultaneously so that the correlation of hand movements to the resultant EUS image could be well understood ( $\mathbf{F i g}$. 1). The teaching was primarily focused on technique of EUS and explanation of different maneuvers, handeye coordination and generation of appropriate images. Multiple repetitions were done of the same manoeuvre for the trainees to understand better ( $\vee$ Video 1 ).

The course curriculum ( $\triangleright$ Table 2 ) included two classes on endoscope handling, cleaning and disinfection, and learning the keyboard controls. This was followed by 12 classes on linear EUS anatomy of mediastinum, pancreatico-biliary region, and 


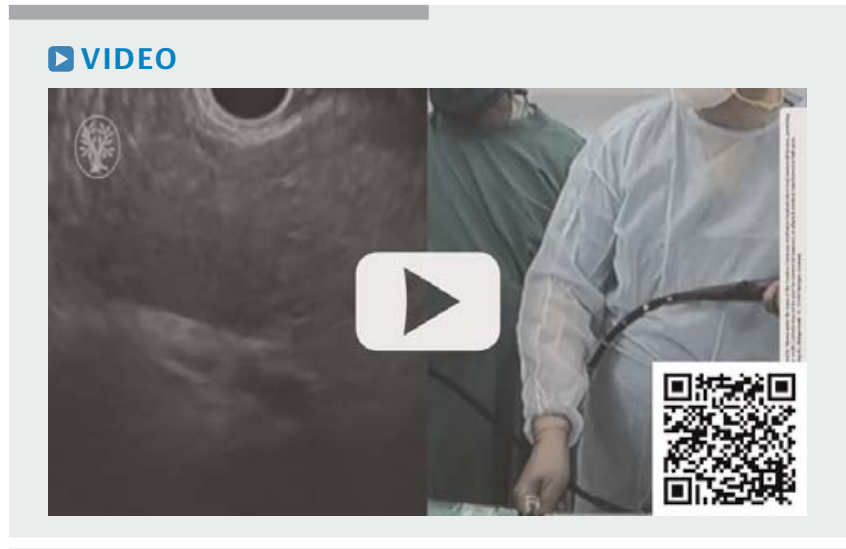

- Video 1 Video of EUS procedure performed by endosonographer showing synchronization of hand movements and resultant EUS image.

rectum. EUS-guided fine-needle aspiration (FNA) was covered in two classes ( $\mathbf{F i g . 2}$ ). Finally, the trainees were briefly exposed to interventional EUS procedures in a stepwise manner in two classes. Questions about technical issues were encouraged during the class, with immediate answers, shown live. At the end of class, 15-minute question-and-answer sessions were held in which problems were solved. A video of the entire class was sent to trainees for review. Trainees were encouraged to

- Table2 Course curriculum: practical classes.

\begin{tabular}{|c|c|c|}
\hline Live cases & $\begin{array}{l}\text { Number } \\
\text { of clas- } \\
\text { ses }\end{array}$ & $\begin{array}{l}\text { Total dura- } \\
\text { tion (min- } \\
\text { utes) }\end{array}$ \\
\hline $\begin{array}{l}\text { Details of radial and linear echo-endo- } \\
\text { scopes and handling of scope }\end{array}$ & 1 & 90 \\
\hline $\begin{array}{l}\text { Cleaning, disinfection and learning the } \\
\text { keyboard controls }\end{array}$ & 1 & 90 \\
\hline Basics of linear EUS and radial EUS & 1 & 90 \\
\hline Linear EUS anatomy of mediastinum & 2 & 180 \\
\hline Railroad technique and alpha maneuver & 2 & 270 \\
\hline Pancreaticobiliary region & 4 & 360 \\
\hline Rectal anatomy & 1 & 90 \\
\hline $\begin{array}{l}\text { EUS-guided tissue sampling techniques: } \\
\text { FNA and FNB }\end{array}$ & 2 & 180 \\
\hline $\begin{array}{l}\text { Interventional EUS exposure } \\
\text { - EUS-guided biliary drainage } \\
\text { - EUS-guided hepaticogastrostomy } \\
\text { - EUS-guided rendezvous } \\
\text { - EUS-guided pseudocyst drainage } \\
\text { - EUS-guided glue \& coiling } \\
\text { " EUS-guided celiac plexus } \\
\text { - EUS-guided RFA ablation } \\
\text { - EUS-guided gastrojejunostomy }\end{array}$ & 2 & 180 \\
\hline $\begin{array}{l}\text { EUS, endoscopic ultrasound; FNA, fine-need } \\
\text { biopsy. }\end{array}$ & t. & fine-needle \\
\hline
\end{tabular}

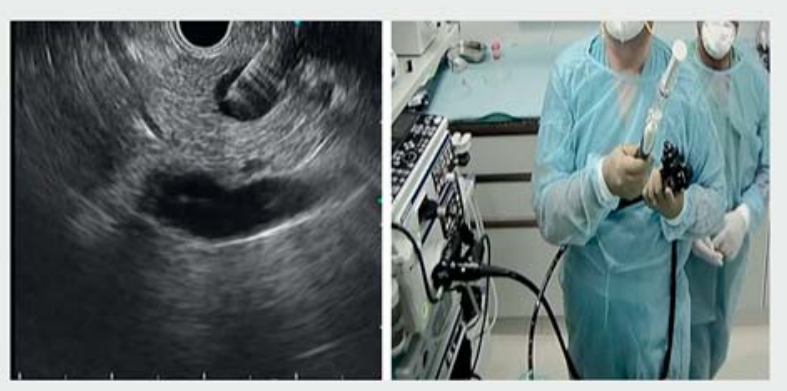

Fig. 2 Virtual training screen of two equal images, one image showing examiner's hand movements while taking tissue sampling with echo-endoscope and another image is showing EUS picture.

ask questions in the interim period and send video of their own procedures done at their institute.

The assessment included a questionnaire after each class, a video assessment after each class in which the trainees would do the procedure and send us a video, and a final questionnaire at the end of the course. At all stages after each class, the recorded videos were assessed by an expert who identified mistakes and technical difficulties during the procedure and also corrected them in the next session virtually.

Objective end points were assessed by a video assessment of satisfactory identification of specific structures. Objective performance assessment was done on the following end points: 1 . passage of scope till second part of duodenum; 2. identifying the structures - a) sub-carinal space, b) head of pancreas, c) common bile duct, d) body and Ttil of pancreas; and 3. ability to get appropriate windows. Trainee competency was assessed by the mentor. There was no intentional pre-selection of videos by trainees or the mentor. All the videos sent by the trainees were continuously assessed and feedback was given. Area identification was defined as ability to identify a specific organ by its echo-texture and anatomical relations. We differentiated the ability to identify a structure from getting an appropriate window by the trainee's ability to visualize the specific area completely without artefacts, and to generate images which appeared like standard image of that organ (textbook image) over a period of time, and not just in one frame. This description has been added in the methods section

\section{Results}

A total of 21 trainees (14 males, 7 females) from nine countries (India, Pakistan, Bangladesh, Myanmar, Paraguay, Colombia, United States, UAE, and Lebanon) participated in the course. Pre-course, four trainees had done less than 50 cases, 14 trainees had exposure to EUS anatomy via short EUS training courses, and three trainees had no exposure other than videos and books. Twenty trainees (95\%) had prior ERCP experience. All 21 trainees attended all the classes or went through the video recording of the class. All trainees returned the weekly questionnaires. Fifteen trainees $(71 \%)$ sent a video for the final assessment ( $\triangleright$ Video 2 ). 


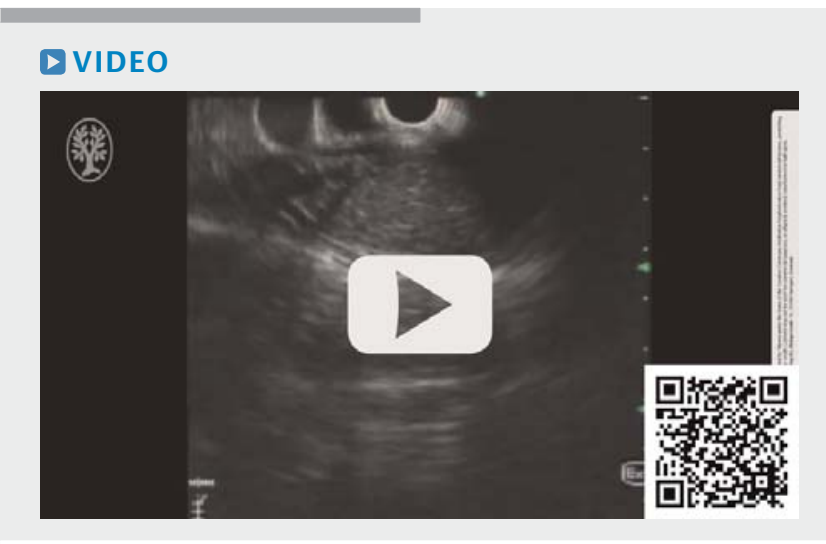

- Video 2 Recorded video of EUS procedure performed by trainee showing common bile duct.

\section{Subjective assessment}

Twenty of 21 trainees performed 251 EUS procedures (range 825 , mean $12.5 \pm 4.9$ ) at their institutions. All of them reported satisfaction with the classes and improvement in their technical capabilities. One trainee did not do procedures at his institute.

\section{Objective assessment}

Objective assessment as per predefined criteria was available for 15 trainees.

\section{Passage of scope}

All students (100\%) could maneuver the echo-endoscope through the cricopharynx, esophagus, stomach and duodenum.

\section{Identification of specified areas}

The successful area identification rates were 12 of 15 (80\%) for the subcarinal space, 10 of $15(66.6 \%)$ for the head of the pancreas, 10 of 15 (66.6\%) for the common bile duct, and nine of $15(60 \%)$ for the tail of the pancreas ( Fig. 3 ).

\section{Ability to get appropriate windows}

The success rate was 10 of 15 (67\%) for the subcarinal space, eight of 15 (53.3\%) for the head of the pancreas, seven of 15 (46.6\%) for the common bile duct and six of 15 (40\%) for the tail of the pancreas.

\section{Adverse events}

There were no perforations or other adverse events reported ( Table 3).

\section{Discussion}

To the best of our knowledge, this is the first study to assess the feasibility of structured EUS training in a virtual EUS course. The possibility of virtual training during the COVID-19 pandemic has been mentioned, but has not been prospectively evaluated [2]. We found that virtual training is safe, can impart training in
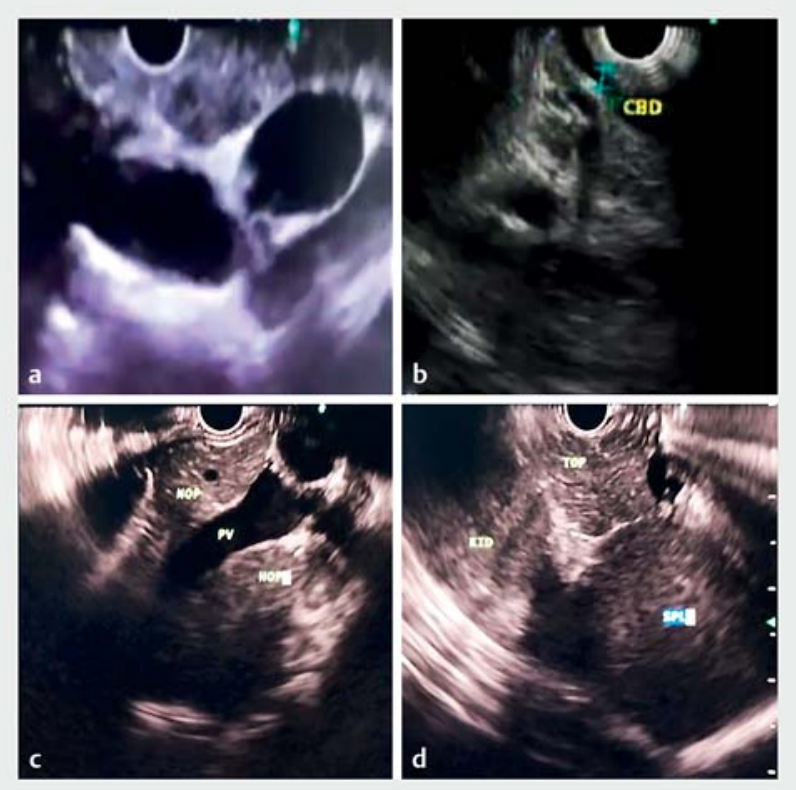

- Fig. 3 EUS images from procedures done by students at their institute. a Subcarinal lymph node. b Common bile duct done. c Head of pancreas. d Tail of pancreas.

$\checkmark$ Table 3 Objective assessment of trainees.

\begin{tabular}{|l|l|}
\hline Questionnaires & 21 \\
\hline Trainees who performed EUS at their institute & 20 \\
\hline Video assessment & 15 \\
\hline Objective end points & \\
\hline Video assessment & \\
\hline - Passage of echo-endoscope & $15 / 15(100 \%)$ \\
\hline Successful area identification rates & \\
\hline - Subcarinal space & $12 / 15(80 \%)$ \\
\hline - Head of pancreas & $10 / 15(66.6 \%)$ \\
\hline - Common bile duct & $10 / 15(66.6 \%)$ \\
\hline - Tail of pancreas & $9 / 15(60 \%)$ \\
\hline Ability to get appropriate windows & \\
\hline - Subcarinal space & $10 / 15(67 \%)$ \\
\hline - Head of pancreas & $8 / 15(53.3 \%)$ \\
\hline - Common bile duct & $7 / 15(46.6 \%)$ \\
\hline - Tail of pancreas & $6 / 15(40 \%)$ \\
\hline Adverse events & 0 \\
\hline EUS, endoscopic ultrasound. & \\
\hline
\end{tabular}


multiple countries simultaneously, and has good acceptability among trainees. There was evidence of both technical and cognitive improvement at the end of the 3-month training period. The classes were held mainly once a week over a weekend, thus not hampering the regular schedule of the trainees.

Performing EUS competently requires technical and cognitive abilities beyond the scope of conventional endoscopy and it needs a long learning curve. One-on-one hands-on training during fellowship is the gold standard for training in EUS. But fellowships are scarce and not easily available. In the United States, there have been approximately 100 applicants per year and 60 participating programs for advanced endoscopy fellowships [3]. Each year, about $20 \%$ of graduating gastroenterology fellows apply for advanced endoscopy fellowship, and applicant match rates are approximately $60 \%$. This creates a high demand among gastroenterology fellows to learn EUS with a non-fellowship approach. In Asia even fewer fellowships are available, with an Asia-Pacific survey in 2004 showing that only $22.5 \%$ of the practicing endosonographers had a formal overseas fellowship lasting at least 6 months and $49.3 \%$ of EUS practitioners declared they were self-taught [4].

The average advanced endoscopy trainee needs at minimum 225 EUS procedures in order to achieve core competence, with approximately a $50 \%$ greater number of procedures required in some cases [5]. European Society of Gastrointestinal Endoscopy (ESGE) Technical Guidelines recommend that a minimum of 20 and 30 supervised EUS-FNA of non-pancreatic and pancreatic lesions, respectively, be performed with rapid on-site evaluationbefore assessment of competency in these techniques [6].

Some gastroenterologists start clinical practice without prior EUS experience and wish to learn EUS mid-career. The route of fellowships is not available to them. Quite a few of them start EUS after limited observation or watching videos on internet [4]. There is a need for additional learning techniques before a trainee starts one-on-one hands-on fellowship. It is possible that with prior exposure to training on in vitro or ex vivo models or on virtual platforms, the number of procedure required to achieve competency could be reduced [7]. A structured EUS training program on in vitro models improved EUS skills significantly and post-training, trainees recorded significant improvement in the overall mean (SD) scores in tests conducted (77.5 0.2\% vs. $66.00 .3 \%$ pre-training, $P<0.0001$ ) [8]. Barthet et al reported that teaching EUS with a live pig model significantly increased competence in diagnostic procedures with regard to visualizing anatomical structures and performance of FNA [9]. The addition of these methods prior to handson training could possibly reduce the number of cases required for competence.

Virtual simulators are available (GI Mentor II, Symbionix Corp, United States), although there are issues with tactile and haptic feedback. In addition, the virtual training models are not actual patients. Virtual training on patients with live streaming is an attractive concept. It allows training on live patients at a distance with active mentoring regarding the procedural technical details. If performed by experienced mentors, the risks are negligible, and appropriate images with simultaneous EUS and mentor's hand movements could impart learning quickly. The availability of echo-endoscopes at the trainee's institution allows for practice in their own environment on their own patients. Regular feedback is possible via video recordings, and technical deficiencies can be identified and corrected. We evaluated this approach to overcome the shortage of training opportunities during the COVID-19 pandemic.

Our study had trainees from different countries and different levels of EUS training but none of them had been trained adequately in EUS. We found that the skill of passing the scope till duodenum was the easiest to learn. None of our trainees reported a perforation. The skill to identify various organs took a relatively longer time. Identifying the carina was understandably easy, while it was difficult to identify the common bile duct and the tail of the pancreas. The most difficult part was to get appropriate windows for specific areas. We do not expect these trainees to get appropriate windows after watching just 21 procedures, but it was heartening to see that trainees could get a good window for the subcarinal space, and a smaller number could get windows for the common bile duct and the tail of the pancreas.

Our results indicate that virtual live training has the potential to become an important initial step in learning EUS. Trainees appear to understand the organ identification relatively easily, and the hand-eye coordination probably improves after watching the mentor's movements and resultant images. Smaller faults like continuous instillation of air, excessive rotational body movements, and basic scope positions can be easily rectified after watching their videos. Continuous emphasis on making small measured movements improves the image quality and getting the appropriate windows.

Virtual live training has few problems. Trainees perform procedures without mentor supervision, and there could be adverse events like perforation. We attempted to minimize this problem by including only trainees with adequate experience in diagnostic procedures. In addition, the requirement of availability of echo-endoscope in their institution ensures that there is a well-run EUS service at their centers. All the trainees did not send video feedbacks. Another issue is the lack of well-defined objective criteria to assess competence. Although we gave them questionnaires at the end of each session and video recordings of procedures done by trainees were available, it is still desirable to have better objective criteria for competence measurement during remote training.

It is obvious that a virtual training course cannot be a substitute for one-on-one hands-on training, but it could serve as an important initial step in preparing the trainees before the advanced endoscopy fellowship. Virtual live training could also become an important integral part of the fellowship course. The possibility of training large number of trainees from multiple countries could also make it useful for national endoscopy societies to run educational courses.

\section{Conclusions}

This feasibility study demonstrates that virtual live training is safe and provides enough initial theoretical and technical information to trainees to understand the basics of EUS procedure. 
Incorporation of virtual training into regular fellowship programs could possibly hasten the learning process. Objective parameters for trainee competence assessment during virtual training need to be developed.

\section{Competing interests}

The authors declare that they have no conflict of interest.

\section{References}

[1] Faulx AL, Lightdale JR, Acosta RD et al. Guidelines for privileging, credentialing, and proctoring to perform Gl endoscopy. Gastrointest Endosc 2017; 85: 273-281

[2] Siddiqui UD, Aslanian HR. The new virtual reality: advanced endoscopy education in the COVID-19 era. Digest Dis Sci 2020; 65: 18881891

[3] Wani S, Keswani RN, Petersen B et al. Training in EUS and ERCP: standardizing methods to assess competence. Gastrointest Endosc 2018; 87: $1371-1382$
[4] Ho KY. Survey of endoscopic ultrasonographic practice and training in the Asia-Pacific region. J Gastroenterol Hepatol 2006; 21: 1231-1235

[5] Wani S, Han S, Simon V et al. Setting minimum standards for training in EUS and ERCP: results from a prospective multicenter study evaluating learning curves and competence among advanced endoscopy trainees. Gastrointest Endosc 2019; 89: 1160-1168

[6] Polkowski M, Larghi A, Weynand B et al. Learning, techniques, and complications of endoscopic ultrasound (EUS)- guided sampling in gastroenterology: European Society of Gastrointestinal Endoscopy (ESGE) Technical Guideline. Endoscopy 2012; 44: 190-206

[7] Dhir V, Itoi T, Fockens P et al. Novel ex vivo model for handson teaching of and training in EUS-guided biliary drainage: creation of "Mumbai EUS" stereolithography/3D printing bile duct prototype (with videos). Gastrointest Endosc 2015; 81: 440-446

[8] Wang MH, Dy F, Vu VK et al. Structured endoscopic ultrasonography (EUS) training program improved knowledge and skills of trainees: results from the Asian EUS Group. Dig Endosc 2015; 27: 687-691

[9] Barthet M, Gasmi M, Giovannini M et al. EUS training in a live pig model: does it improve echoendoscope hands-on and trainee competence? Endoscopy 2007; 39: 535-539 